\title{
Predictive biomarkers for chemotherapies in pancreatic cancer
}

\author{
Augustin Catalin Dima ${ }^{1,2}$, Alina Dima ${ }^{1}$, Sever Calin Moldovan ${ }^{3}$, Mircea Ciurea $^{1}$
}

Corresponding author: Augustin Catalin DIMA

Dr Carol Davila Central University Emergency Military Hospital,

Bucharest, Romania

88 Mircea Vulcanescu Street,

Bucharest, Romania

E-mail: gustidima@yahoo.com

\author{
"'Carol Davila" University of Medicine and Pharmacy, Bucharest, Romania \\ 2"Dr Carol Davila" Central University Emergency Military Hospital, Bucharest, Romania \\ "University North of France, Free Faculty of Medicine, The Catholic Institute of Lille Hospital Group, \\ Saint Vincent de Paul Hospital, Visceral and Digestive Surgery Department, Lille, France
}

\section{ABSTRACT}

Pancreatic cancer is associated with high rates of mortality especially due to advanced stages of diagnosis. Adjuvant chemotherapy role is thus essential in the attempt to downstage the tumoral grade. In cases of locally advanced tumors, this step could be followed by curative surgical resection. Characterization of predictor biomarkers for adjuvant chemotherapy has drawn increasing interest in molecular biology research of pancreatic cancer. Personalized treatment is one of the solutions proposed for advancing towards a better outcome. In this paper we discuss available predictor biomarkers with focus upon gemcitabine and FOLFIRINOX (5-fluoro uracil (5-FU), irinotecan and oxaliplatin) chemotherapy regimens, present approaches in advanced pancreatic cancer. Many data are current available in relation with gemcitabine chemotherapy regimen, where biomarkers like ribonucleotide reductase large subunit (RRM1), human equilibrative nucleoside transporter-1 (hENT1), deoxycytidine kinase ( $\mathrm{dCK})$, human antigen $\mathrm{R}(\mathrm{HuR})$, and secreted protein acidic rich in cysteine (SPARC) were proposed. Regarding the response to FOLFIRINOX components, other markers such as thymidylate synthethase (TS), topoisomerase I, and excision repair cross-complementation 1 (ERCC1) or KRAS mutation status were also investigated.

Key words: pancreatic cancer, chemotherapy, response predictor, biomarker

\section{BACKGROUND}

Pancreatic cancer is the fourth leading cause of cancer-related mortality (1) in developed countries and it is expected to become the second one (2). Increased mortality in this pathology is mainly related to the advanced stages at diagnosis, cases in which practically the moment for curative resection has been already exceeded (3).

A predictive biomarker is a specific biologic feature associated with the likely probability of benefit with treatment (i.e. chemotherapy). Starting from evidence obtained in other types of cancer, it was formulated the hypothesis of increasing chemotherapy's efficiency in pancreatic adenocarcinoma by the use of predictor biomarkers in an attempt to anticipate the response to adjuvant 
therapy (4). Hence, the development of predictive biomarkers for the characterization of specific subsets of patients in pancreatic cancer is one of the important issues in current consensus reports on pancreatic cancer (5).

We intend to shortly review here the research findings on the influence of predictor biomarkers upon response to treatment of the main adjuvant therapies used for advanced pancreatic cancer, gemcitabine regimen and FOLFIRINOX (Folinic Acid, 5- fluoro uracil (5-FU), Irinotecan, and Oxaliplatin) regimen chemotherapy. We started our work with a Medline search using related keywords (i.e. pancreatic cancer and predictive biomarker, gemcitabine, irinotexan, oxaliplatin, 5-FU) with focus on data related to clinical data.

\section{GEMCITABINE RESPONSE PREDICTORS}

Gemcitabine is a nucleoside analogous used in adjuvant chemotherapy for pancreatic cancer. The efficiency of gemcitabine is not guaranteed when applied in unselected pancreatic cancer patient as resistance to gemcitabine may occur in some of the treated patients also (6).

The ribonucleotide reductase large subunit (RRM1) participates to the regulatory processes of cell proliferation, migration and also synthesis of deoxyribonucleotides, hence it can be a possible important component in tumor growth process. The RRM1 is actually possible cellular target for gemcitabine and its value as predictor for the response to this chemotherapy was studied in different neoplasia such as lung, breast, biliary tract, pancreatic cancer included (7). The RRM1 is actually an intracellular target for gemcitabine, so it was proposed as useful biomarker in pancreatic cancer (8).

The levels of RRM1, human equilibrative nucleoside transporter-1 (hENT1) or deoxycytidine kinase $(\mathrm{dCK})$, were not shown to be predictive for survival in pancreatic cancer patients without adjuvant chemotherapy (9). But, in patients treated with gemcitabine, the presence of elevated in situ RRM1 expression was associated with significantly better overall survival than in patients with low RRM1 (6). The research results are however not uniform, since there are some other authors that did not approve the RRM1 use as a predictive biomarker in pancreatic cancer (10).

\section{Human equilibrative nucleoside trans- porter-1 (hENT1)}

The nucleoside-derived molecules like gemcitabine need transmembranary transportation in order to transform into an active drug. This explains how hENT over expression produces an enhanced gemcitabine action in vivo (11).

According to its properties, the nucleoside transporter hENT1 is one of the predictor biomarkers in the pancreatic cancer (8). Upon multivariate analysis models, both hENT1 and dCK levels were independent predictors of survival after gemcitabine treatment (9). Also, both hENT1 and human nucleoside transporter 3 levels seem to be useful in predicting survival in pancreatic cancer patients treated by gemcitabine (12). On the contrary, the hENT1 expression in pancreatic cancer advanced metastasis is not associated with overall survival after pancreatic cancer (13). Other authors reported that the correlations made between hENT1 and survival in pancreatic cancer (i.e. low expression, associated with worse outcome) is independent of gemcitabine therapy (14).

In a prospective randomized trial that included 538 patients with pancreatic cancer, the increased hENT1 presence was associated with better outcome, overall and disease-free survival only in those undergoing gemcitabine therapies, but not when chemotherapy with 5-FU was applied (15). These data outlined the need for personalized treatment in pancreatic cancer (16), but still, obtaining an accurate method for hENT1 determination in daily practice requires further research (17). It was so also inferred that in pancreatic cancer, the gemcitabine regimen should not be indicated as adjuvant chemotherapy in patients with low hENT1 expression (18).

\section{Deoxycytidine kinase (dCK) and human antigen $\boldsymbol{R}(\boldsymbol{H u R})$}

Human antigen R (HuR) is a RNA-binding protein associated with $\mathrm{dCK}$ involved in the metabolization followed by activation of the gemcitabine molecule. The HuR over expression leads to enhanced dCK protein presentation and plays an important role in the gemcitabine effect upon the tumor cells (19).

The HuR seems to be involved in the regulation of the pancreatic cell phenotype in critical states such as glucose deprivation, in facilitating the cellular survival in an impaired environment (20). The HuR expression is correlated with the tumor $\mathrm{T}$ stage and also a predictor of outcome in resected pancreatic cancers (21).

\section{Secreted protein acidic and rich in cysteine (SPARC)}

Secreted protein acidic and rich in cysteine (SPARC), albumin-binding protein, is associated with cells' migration and differentiation; all of which are processes 
playing a key role in oncogenesis (22). The results of a study that included patients with metastatic pancreatic adenocarcinoma treated with maximum-tolerated doses of gemcitabine plus nab-paclitaxel showed that the SPARC expression was independently associated with the overall survival in multivariate regression models that included as covariates the sex, race, age, treatment, and baseline CA19-9 level. Even so, significant correlation was obtained only for the stromal SPARC and not for the SPARC expression in the tumor cells (23).

In regard with the data presented above, pancreatic tumors with low hENT1 and high RRM1 expression might be considered for chemotherapy regimens that include gemcitabine in future consensus statements. But also, further research is needed in order to validate this information and to improve the knowledge of the other biomarkers proposed as predictors in pancreatic cancer.

\section{FOLFIRINOX}

FOLFIRINOX, chemotherapy regimen including folinic acid, 5-FU, irinotecan and oxaliplatin, is used in advanced pancreatic cancer where a surgical resection is not suitable due to the advanced loco-regional disease. The purpose is to obtain in a neoadjuvant setting the tumoral down-staging in order to increase the pancreatic adenocarcinoma resectability rates.

After proving efficiency in pancreatic cancer (24, $25)$, the FOLFIRINOX was tested versus gemcitabine in a randomized trial with good results on survival rates. However, the improvement on the overall survival was grieved by the cost of increased toxicity (26).

FOLFIRINOX was observed to have greater efficiency in female patients and within the presence of high serum CA 19.9 levels (27). In this respect, it is to be noted that the current use of CA19-9 is for diagnostic and prognostic ends and not as a response predictor biomarker. For the moment, there are no data available on other predictor biomarkers for the FOLFIRINOX regimen, but efforts are done in order to identify targets for the individual chemotherapies with this regimen.

\section{5-FLUORO-URACIL (5-FU) RESPONSE PREDICTORS}

\section{Deoxycytidine kinase (dCK) and human antigen $\boldsymbol{R}(\boldsymbol{H u R})$}

After exploring their utility as predictors of the gemcitabine treatment response, in a randomized clinical trial in which pre-operative samples of pancreatic cancer were analyzed, it was found that the expression of both dCK and HuR is strongly correlated. Moreover, the dCK levels were predictive for the response to 5-FU (28).

\section{Thymidylate synthetase (TS)}

Thymidylate synthetase (TS), enzyme involved in the DNA synthesis processes, is a target in the chemotherapy with 5-FU. In 131 patients with pancreas adenocarcinoma the TS expression was explored in all cases. The rates of survival were similar between patients with low TS expression when compared with those with high TS expression in patients with palliative treatment. On the contrary, after pancreatic resection, the intensive chemotherapy was followed by a better outcome in patients with low TS expression (29). Not only for the pancreatic adenocarcinoma, but also in other cancers, the low TS expression was positively correlated with a better outcome (30).

In another research, the response to S1 - based chemotherapy was tested in the pancreatic cancer (31). The S-1, dihydropyrimidine dehydrogenase inhibitory fluoropyrimidine, is an oral adjuvant chemotherapy obtained by the modulation of 5-FU (32). The TS, dihydropyrimidine dehydrogenase (DPD), and orotate phosphoribosyltransferase expression was searched in 106 patients with pancreatic cancer. Among the three markers, DPD was the best predictor marker for the S1-based chemotherapy (31).

\section{IRINOTECAN RESPONSE PREDICTORS}

Irinotecan is a plant alkaloid classified as a topoisomerase I inhibitor that proved efficient in chemotherapy regimens for advanced pancreatic cancer.

\section{Topoisomerase I}

In patients with metastatic colorectal cancer, irinotecan was administrated when topoisomerase I expression was positive, and oxaliplatin was administrated in patients with negative topoisomerase I and positive ERCC1 expression, but this study did not find significant benefits on the overall survival (33). Still, we do not have yet evidence of the topoisomerase I expression relevance in pancreatic cancer; this will probably be a new research area in pancreatic cancer predictors.

\section{OXALIPLATIN RESPONSE PREDICTORS}

Oxaliplatin (trans-I-1,2-diaminocyclohexane oxalato- 
platinum) is an antineoplastic platinum derivate with a 1,2-diaminocyclohexane carrier ligand (34) that showed anti-neoplastic effects against different pancreatic tumoral cell lines, $\mathrm{PMH} 2 / 89$ and fresh liver metastases secondary to pancreatic neoplasia included (35).

\section{Excision repair cross-complementation 1 (ERCC1)}

The excision repair cross-complementation 1 (ERCC1) is a key regulatory enzyme with expression related to the survival in several cancers (36), pancreatic cancer included. It was also proposed as a response predictor marker for the adjuvant chemotherapy in pancreatic cancer (37). The increased expression of excision repair cross-complementation 1 (ERCC1) in resected pancreatic cancers seems to be associated with lower recurrence-free interval rates and worse overall survival (38).

\section{ERLOTINIB RESPONSE PREDICTORS}

\section{Excision repair cross-complementation 1 (ERCC1)}

The cases in which a higher ERCC1 staining pattern was observed had better responses to the erlotinib associated with the combination gemcitabine - oxaliplatin (39).

\section{KRAS mutation status}

The hypothesis that genetic background could characterize subsets of pancreatic cancer patients sensitive to certain drugs introduced the possibility of personalized chemotherapy in cancer patients according to gene expression (40). The KRAS mutation status in patients with pancreatic cancer treated with erlotinib was found to be related with the overall survival even if not in correlation with the response to the adjuvant chemotherapy (41).

Thus, until now less is now about possible predictor biomarkers in rapport with each therapeutic principle of FORFIRINOX regimen and nothing in regard with a predictor for the response to the entire chemotherapeutic regimen.

\section{CONCLUSIONS}

The knowledge in the molecular biology of the pancreatic cancer is advancing. Hypothesis on the possible treatment options starting from the tumor molecular biology data are starting to be defined. In this respect, it seems that chemotherapy regimens based on gemcitabine do not have efficiency in pancreatic tumors that present low hENT1 expression.

We presented in this paper also researches that has low level of evidence, as in this moment large clinical trials are lacking. There is so certainly need for further research in this field; but there is also hope that the advances in developing personalized treatment strategies will improve the outcome, the overall survival and the disease-free survival in a dreadful disease like the pancreatic adenocarcinoma.

\section{Conflict of interest}

Nothing to report

\section{Source of funding}

Nothing to report

\section{Acknowledgement}

Nothing to report

\section{REFERENCES}

1. http://www.pancreatic.org/site/c.htJYJ8MPIwE/b.5050503/k.40C9/ Pancreatic_Cancer_Facts.htm

2. Chiorean $\overline{E G}$, Coveler AL. Pancreatic cancer: optimizing treatment options, new, and emerging targeted therapies. Drug Des Devel Ther. 2015;9:352945.

3. Lamarca A, Feliu J. Pancreatic biomarkers: could they be the answer? World J Gastroenterol. 2014;20(24):7819-29.

4. Kruger S, Haas M, Ormanns S, Bסchmann S, Siveke JT, Kirchner T, et al. Translational research in pancreatic ductal adenocarcinoma: current evidence and future concepts. World J Gastroenterol. 2014;20(31):1076977.

4. Philip PA, Mooney M, Jaffe D, Eckhardt G, Moore M, Meropol N, et al. Consensus report of the national cancer institute clinical trials planning meeting on pancreas cancer treatment. J Clin Oncol. 2009 Nov 20; 27(33):5660-9.

6. Akita H, Zheng Z, Takeda Y, Kim C, Kittaka N, Kobayashi S, et al. Significance of RRM1 and ERCC1 expression in resectable pancreatic adenocarcinoma. Oncogene. 2009 Aug 13;28(32):2903-9.

7. Jordheim LP, SOve P, Tridan 0 , Dumontet $C$. The ribonucleotide reductase large subunit (RRM1) as a predictive factor in patients with cancer. Lancet Oncol. 2011 Jul;12(7):693-702.

8. Jordheim LP, Dumontet C. Do hENT1 and RRM1 predict the clinical benefit of gemcitabine in pancreatic cancer? Biomark Med. 2013 Aug;7(4):663-71.

9. Marıchal R, Bachet JB, Mackey JR, Dalban C, Demetter P, Graham K, et al. Levels of gemcitabine transport and metabolism proteins predict survival times of patients treated with gemcitabine for pancreatic adenocarcinoma. Gastroenterology. 2012 Sep;143(3):664-74.e1-6.

10. Valsecchi ME, Holdbrook T, Leiby BE, Pequignot E, Littman SJ, Yeo CJ, et al. Is there a role for the quantification of RRM1 and ERCC1 expression in pancreatic ductal adenocarcinoma? BMC Cancer. 2012 Mar 22;12:104.

11. Purez-Torras S, Garcva-Manteiga J, Mercadı E, Casado FJ, Carbo N, PastorAnglada M, et al. Adenoviral-mediated overexpression of human equilibrative nucleoside transporter 1 (hENT1) enhances gemcitabine response in human pancreatic cancer. Biochem Pharmacol. 2008 Aug 1;76(3):322-9.

12. Marıchal R, Mackey JR, Lai R, Demetter P, Peeters M, Polus M, et al. Human equilibrative nucleoside transporter 1 and human concentrative nucleoside transporter 3 predict survival after adjuvant gemcitabine therapy in resected pancreatic adenocarcinoma. Clin Cancer Res. 2009 Apr 15;15(8):2913-9.

13. Poplin E, Wasan H, Rolfe L, Raponi M, Ikdahl T, Bondarenko I, et al. Randomized, multicenter, phase II study of C0-101 versus gemcitabine in patients with metastatic pancreatic ductal adenocarcinoma: including a 
prospective evaluation of the role of hENT1 in gemcitabine or CO-101 sensitivity. J Clin Oncol. 2013 Dec 10;31(35):4453-61.

14. Kim R, Tan A, Lai KK, Jiang J, Wang Y, Rybicki LA, et al. Prognostic roles of human equilibrative transporter 1 (hENT-1) and ribonucleoside reductase subunit M1 (RRM1) in resected pancreatic cancer. Cancer. 2011 Jul 15;117(14):3126-34.

15. Farrell JJ, Elsaleh $\mathrm{H}$, Garcia M, Lai R, Ammar A, Regine WF, et al. Human equilibrative nucleoside transporter 1 levels predict response to gemcitabine in patients with pancreatic cancer. Gastroenterology. 2009 Jan;136(1): 187-95.

16. Ko AH, Tempero MA. Personalized medicine for pancreatic cancer: a step in the right direction. Gastroenterology. 2009 Jan;136(1):43-5.

17. Surcek M, Cros J, Marıchal R, Bachet JB, Fljou JF, Demetter P. Human equilibrative nucleoside transporter 1 testing in pancreatic ductal adenocarcinoma: a comparison between murine and rabbit antibodies. Histopathology. 2015 Feb;66(3):457-62.

18. Greenhalf W, Ghaneh P, Neoptolemos JP, Palmer DH, Cox TF, Lamb RF, et al; European Study Group for Pancreatic Cancer. Pancreatic cancer hENT1 expression and survival from gemcitabine in patients from the ESPAC-3 trial. J Natl Cancer Inst. 2014 Jan;106(1):dit347.

19. Costantino CL, Witkiewicz AK, Kuwano Y, Cozzitorto JA, Kennedy $E P$, Dasgupta $A$, et al. The role of HuR in gemcitabine efficacy in pancreatic cancer: HuR Up-regulates the expression of the gemcitabine metabolizing enzyme deoxycytidine kinase. Cancer Res. 2009 Jun 1; 69(11):4567-72.

20. Burkhart RA, Pineda DM, Chand SN, Romeo C, Londin ER, Karoly ED, et al. HuR is a post-transcriptional regulator of core metabolic enzymes in pancreatic cancer. RNA Biol. 2013 Aug;10(8):1312-23.

21. Richards NG, Rittenhouse DW, Freydin B, Cozzitorto JA, Grenda D, Rui H, et al. HuR status is a powerful marker for prognosis and response to gemcitabine-based chemotherapy for resected pancreatic ductal adenocarcinoma patients. Ann Surg. 2010 Sep;252(3):499-505.

22. Yiu GK, Chan WY, Ng SW, Chan PS, Cheung KK, Berkowitz RS, et al. SPARC (secreted protein acidic and rich in cysteine) induces apoptosis in 0 varian cancer cells. Am J Pathol. 2001 Aug;159(2):609-22.

23. Von Hoff DD, Ramanathan RK, Borad MJ, Laheru DA, Smith LS, Wood $T E$, et al. Gemcitabine plus nab-paclitaxel is an active regimen in patients with advanced pancreatic cancer: a phase I/II trial. J Clin Oncol. 2011 Dec 1;29(34):4548-54.

24. Ychou M, Conroy T, Seitz JF, Gourgou S, Hua A, Mery-Mignard D, et al. An open phase I study assessing the feasibility of the triple combination: oxaliplatin plus irinotecan plus leucovorin/ 5-fluorouracil every 2 weeks in patients with advanced solid tumors. Ann Oncol. 2003 Mar; 14(3):481-9.

25. Conroy T, Paillot B, Frannois E, Bugat R, Jacob JH, Stein U, et al. Irinotecan plus oxaliplatin and leucovorin-modulated fluorouracil in advanced pancreatic cancer - a Groupe Tumeurs Digestives of the Federation Nationale des Centres de Lutte Contre le Cancer study. J Clin Oncol. 2005 Feb 20;23(6):1228-36.

26. Conroy T, Desseigne F, Ychou M, Bouchı O, Guimbaud R, Bıcouarn Y, et $\mathrm{al}$; Groupe Tumeurs Digestives of Unicancer;PRODIGE Intergroup. FOLFIRINOX versus gemcitabine for metastatic pancreatic cancer. N Engl $J$ Med. 2011 May 12;364(19):1817-25.

27. Hohla F, Hopfinger G, Romeder F, Rinnerthaler G, Bezan A, Stottner S, et al. Female gender may predict response to FOLFIRINOX in patients with unresectable pancreatic cancer: a single institution retrospective review. Int J Oncol. 2014 Jan;44(1):319-26.

28. McAllister F, Pineda DM, Jimbo M, Lal S, Burkhart RA, Moughan J, et al. dCK expression correlates with 5 -fluorouracil efficacy and HuR cytoplasmic expression in pancreatic cancer: a dual-institutional follow-up with the RTOG 9704 trial. Cancer Biol Ther. 2014 Jun 1;15(6):688-98.

29. Formentini A, Sander S, Denzer S, Straeter J, Henne-Bruns D, Kornmann M. Thymidylate synthase expression in resectable and unresectable pancreatic cancer: role as predictive or prognostic marker? Int J Colorectal Dis. 2007 Jan;22(1):49-55.

30. Sulzyc-Bielicka V, Domagala P, Bielicki D, Safranow K, Domagala W. Thymidylate synthase expression and p21(WAF1)/p53 phenotype of colon cancers identify patients who may benefit from 5 -fluorouracil based therapy. Cell Oncol (Dordr). 2014 Feb;37(1):17-28.

31. Kondo N, Murakami $\mathrm{Y}$, Uemura $\mathrm{K}$, Sudo $\mathrm{T}$, Hashimoto $\mathrm{Y}$, Nakashima $\mathrm{A}$, Ohge $\mathrm{H}$, et al. Prognostic impact of dihydropyrimidine dehydrogenase expression on pancreatic adenocarcinoma patients treated with S-1-based adjuvant chemotherapy after surgical resection. J Surg Oncol. 2011 Aug 1;104(2):146-54.

32. Maehara Y. S-1 in gastric cancer: a comprehensive review. Gastric Cancer. 2003;6 Suppl 1:2-8.

33. Cubillo A, Rodriguez-Pascual J, Lopez-Rvos F, Plaza C, Garcva E, Alvarez $\mathrm{R}$, et al. Phase II Trial of Target-guided Personalized Chemotherapy in Firstline Metastatic Colorectal Cancer. Am J Clin Oncol. 2014 Feb 10.

34. Raymond E, Chaney SG, Taamma A, Cvitkovic E. Oxaliplatin: a review of preclinical and clinical studies. Ann Oncol. 1998 Oct;9(10):1053-71.

35. Kornmann M1, Fakler H, Butzer U, Beger HG, Link KH. Oxaliplatin exerts potent in vitro cytotoxicity in colorectal and pancreatic cancer cell lines and liver metastases. Anticancer Res. 2000 Sep-0ct;20(5A):3259-64.

36. Squires MH 3rd, Fisher SB, Fisher KE, Patel SH, Kooby DA, El-Rayes BF, et al. Differential expression and prognostic value of ERCC1 and thymidylate synthase in resected gastric adenocarcinoma. Cancer. 2013 Sep 1;119(17):3242-50.

37. Maithel SK, Coban I, Kneuertz PJ, Kooby DA, El-Rayes BF, Kauh JS, et al. Differential expression of ERCC1 in pancreas adenocarcinoma: high tumor expression is associated with earlier recurrence and shortened survival after resection. Ann Surg Oncol. 2011 Sep;18(9):2699-705.

38. Fisher SB, Patel SH, Bagci P, Kooby DA, El-Rayes BF, Staley CA 3rd, et al. An analysis of human equilibrative nucleoside transporter-1, ribonucleoside reductase subunit M1, ribonucleoside reductase subunit M2, and excision repair cross-complementing gene- 1 expression in patients with resected pancreas adenocarcinoma: implications for adjuvant treatment. Cancer. 2013 Jan 15;119(2):445-53.

39. Fuereder T, Stift J, Kuehrer I, Stranzl N, Hoeflmayer D, Kornek G, et al. Response to GEMOX plus erlotinib in pancreatic cancer is associated with ERCC1 overexpression. Eur J Clin Invest. 2014 0ct:44(10):958-64.

40. Cui Y, Brosnan JA, Blackford AL, Sur S, Hruban RH, Kinzler KW, Vogelstein $B$ et al. Genetically defined subsets of human pancreatic cancer show unique in vitro chemosensitivity. Clin Cancer Res. 2012 Dec 1;18(23): 6519-30.

41. Boeck S, Jung A, Laubender RP, Neumann J, Egg R, Goritschan C, et al. KRAS mutation status is not predictive for objective response to anti-EGFR treatment with erlotinib in patients with advanced pancreatic cancer. J Gastroenterol. 2013 Apr;48(4):544-8. 